\title{
Cross-ratio inequality with infinite type of singularities
}

\section{Sokhobiddin Akhatkulov ${ }^{*}$ and Mohd Salmi Md Noorani}

\section{"Correspondence:}

akhatkulov@yahoo.com

School of Mathematical Sciences,

Faculty of Science and Technology,

University Kebangsaan Malaysia,

43600 UKM, Bangi, Selangor Darul

Ehsan, Malaysia

\begin{abstract}
Let $f$ be a preserving orientation circle homeomorphism with infinite number of break points, i.e., the points at which the derivative of $f$ has jumps, and finite number of singular points, i.e., the points $x_{i}, i=1,2, \ldots, n$, such that $f^{\prime}\left(x_{i}\right)=\infty, i=1,2, \ldots, n$. Then the cross-ratio inequality with respect to $f$ holds.
\end{abstract}

MSC: 37E10; 37C15

Keywords: cross-ratio inequality; critical circle homeomorphism; break point; rotation number

\section{Introduction}

Let $S^{1}=\mathbf{R} / \mathbf{Z}$ with clearly defined orientation, metric, Lebesgue measure and the operation of addition be the unit circle. Let $\pi: \mathbf{R} \rightarrow S^{1}$ denote the corresponding projection mapping that 'winds' a straight line on the circle. An arbitrary homeomorphism $f$ that preserves the orientation of the unit circle $S^{1}$ can be 'lifted' on the straight line $\mathbf{R}$ in the form of the homeomorphism $F: \mathbf{R} \rightarrow \mathbf{R}$ with property $F(x+1)=F(x)+1$ that is connected with $f$ by relation $\pi \circ F=f \circ \pi$. This homeomorphism $F$ is called the lift of the homeomorphism $f$ and is defined up to an integer term. The most important arithmetic characteristic of the homeomorphism $f$ of the unit circle $S^{1}$ is the rotation number

$$
\rho(f)=\lim _{n \rightarrow \infty} \frac{F^{n}(x)}{n} \bmod 1
$$

where $F$ is the lift of $f$ with $S^{1}$ to $\mathbf{R}$. Here and below, for a given map $F, F^{n}$ denotes its $n$th iterate. The rotation number is rational if and only if $f$ has periodic points (see [1]). We say two circle maps $f$ and $g$ are topologically conjugate if there exists a homeomorphism $\varphi$ : $S^{1} \rightarrow S^{1}$ such that $f=\varphi^{-1} \circ g \circ \varphi$. The map $\varphi$ is called conjugating map or just conjugation. If $f$ and $g$ are conjugate, many of their properties are the same, e.g., the possible types of the orbits of the points of $S^{1}$ under $f$ and under $g$ are the same, the rotation number of $f$ is equal to that of $g$, etc.

Early results about the existence of conjugacy to the linear rotation $f_{\rho}: x \rightarrow x+\rho \bmod 1$ are the following theorems.

Poincaré's theorem [2] Let $f$ be a circle homeomorphism with irrational rotation number $\rho$. Then $f$ is semi-conjugate to the rotation $f_{\rho}$.

๑ 2015 Akhatkulov and Md Noorani; licensee Springer. This is an Open Access article distributed under the terms of the Creative Commons Attribution License (http://creativecommons.org/licenses/by/4.0), which permits unrestricted use, distribution, and reproduction in any medium, provided the original work is properly credited. 
Denjoy's theorem [3] A circle diffeomorphism $f$ with irrational rotation number $\rho$ and $\log D f$ of bounded variation is topologically conjugate to the linear rotation $f_{\rho}$.

Denjoy's result has been extended for different classes of circle homeomorphisms. Indepth results have been found, see [4-8]. Two well-known classes of these are the following.

P-homeomorphisms. These are orientation preserving circle homeomorphisms $f \operatorname{dif}-$ ferentiable except in many countable points called break points admitting left and right derivatives (denoted by $f_{-}^{\prime}$ and $f_{+}^{\prime}$ respectively) such that

- there exist some constants $0<\gamma<\zeta<\infty$ such that $\gamma<f^{\prime}(x)<\zeta$ for all $x \in S^{1} \backslash B P(f)$, $\gamma<f_{+}^{\prime}(b)<\zeta$ and $\gamma<f_{-}^{\prime}(b)<\zeta$ for all $b \in B P(f)$, where $B P(f)$ denotes the set of the break points of $f$;

- $\log f^{\prime}$ has bounded variation. In this situation $\log f^{\prime}, \log f_{-}^{\prime}, \log f_{+}^{\prime}$ and $\log \left(f^{-1}\right)^{\prime}$, $\log \left(f_{-}^{-1}\right)^{\prime}, \log \left(f_{+}^{-1}\right)^{\prime}$ have the same total variation denoted by $V=\operatorname{Var} \log f^{\prime}$.

The ratio $\sigma_{f}(b)=\left(f_{-}(b)\right)^{\prime} /\left(f_{+}(b)\right)^{\prime}$ is called jump ratio of $f$ in $b$.

Critical circle homeomorphisms. These are orientation preserving $C^{1}$ circle maps $f$ such that for each $x_{0} \in S^{1}$ there exist $\alpha \geq 1$, a neighborhood $U\left(x_{0}\right)$ of $x_{0}$, and a homeomorphism $\phi: U\left(x_{0}\right) \rightarrow R$ such that $\phi\left(x_{0}\right)=0$, and if $\alpha>1$ then $f(x)= \pm|\phi(x)|^{\alpha}+f\left(x_{0}\right), \forall x \in U\left(x_{0}\right)$ if $\alpha=1$ then $f(x)=|\phi(x)|^{\alpha}+f\left(x_{0}\right), \forall x \in U\left(x_{0}\right)$.

For the class of $P$-homeomorphisms, the classical result of Denjoy can be easily extended, the existence of the conjugating map for this class was proved by Herman in [5]. The existence of the conjugating map for the class of critical real analytic circle maps was proved by Yoccoz [8] and extended by Świątek [6, 7]. In these works the existence of conjugation was shown by estimating cross-ratio distortions (i.e., proving cross-ratio inequality). Note that the cross-ratio distortions were used in dynamical systems for the first time by Yoccoz [8]. The cross-ratio distortions are the most powerful tools to study the existence and smoothness of a conjugating map for the critical circle homeomorphisms.

Our aim in this work is to prove the cross-ratio inequality for a new class of circle homeomorphisms, which will be defined below with the aid of the above two classes and to show the existence of a conjugating map for this new class. We shall talk about the cross-ratio distortions in the next section.

Now we define the new class of circle homeomorphisms as follows.

(i) $f$ is a preserving orientation circle homeomorphism on $S^{1}$.

(ii) There are points $x_{i} \in S^{1}$ and $\alpha_{i} \in(0,1), i=1,2, \ldots, n$, such that $f(x)=\left(x-x_{i}\right)^{\alpha_{i}}+f\left(x_{i}\right)$ for some $\epsilon_{i}$-neighborhoods of each $x_{i}$.

(iii) $f$ is a $P$-homeomorphism on $S^{1} \backslash \bigcup_{i=1}^{n} U_{\epsilon_{i}}\left(x_{i}\right)$.

Note that it follows from condition (ii) that $f^{\prime}\left(x_{i}\right)=\infty, i=1,2, \ldots, n$.

\section{Cross-ratio inequality}

Now we equip $S^{1}$ with the usual metric $|x-y|=\inf \{|\widetilde{x}-\tilde{y}|$, where $\tilde{x}, \tilde{y}$ range over the lifts of $x, y \in S^{1}$ respectively\}. Our main analytic tools are ratio and cross-ratio distortions (see [9]). Let $a, b, c, d \in S^{1}$ be the four points of the circle which preserve orientation; that is, $a \prec b \prec c \prec d \prec a$ on the circle. We define the cross-ratio of these points as

$$
\operatorname{Cr}(a, b, c, d)=\frac{|c-b||d-a|}{|b-a||d-c|}
$$


If $f$ is a continuous and monotone function on $S^{1}$, then the distortion of the cross-ratio by this function is defined by

$$
\operatorname{Dcr}(a, b, c, d ; f)=\frac{\operatorname{Cr}(f(a), f(b), f(c), f(d))}{\operatorname{Cr}(a, b, c, d)}
$$

Moreover, if $f$ is a $C^{1}$ diffeomorphism, then taking limit from (1) when $b \rightarrow a$ and $c \rightarrow d$, we get the following distortion:

$$
R(a, d ; f)=\frac{(f(d)-f(a))^{2}}{(d-a)^{2}} \cdot \frac{1}{f^{\prime}(a) f^{\prime}(d)}
$$

Notice that the cross-ratio distortion is multiplicative with respect to composition for two functions $f$ and $g$ on $S^{1}$, that is,

$$
R(a, d ; f \circ g)=R(g(a), g(d) ; f) \cdot R(a, d ; g) .
$$

The main results of this paper are the following theorems.

Theorem 2.1 Letf be a circle homeomorphism with irrational rotation number satisfying conditions (i)-(iii). Consider a system of intervals $\left\{\left(a_{i}, b_{i}\right) \subset S^{1}, i=1,2, \ldots, k\right\}$. Suppose that this system of intervals covers each point of the circle $S^{1}$ at most $p$ times. Then there exists a constant $C_{1}=C_{1}(p, f)$ which depends on $p$ and $f$ such that the following inequality

$$
\prod_{i=1}^{k} R\left(a_{i}, b_{i} ; f\right) \leq C_{1}
$$

holds.

Inequality (3) is called cross-ratio inequality with respect to $f$. As an application of this theorem, we provide the following second result of this paper.

Theorem 2.2 Let $f$ be a circle homeomorphism with irrational rotation number satisfying conditions (i)-(iii) without break points. Then there exists a circle homeomorphism $\varphi$ such that the following equality

$$
\varphi \circ f=f_{\rho} \circ \varphi
$$

holds.

In the proof of the second main theorem, we use the properties of a dynamical partition, and therefore we introduce this concept.

\section{Dynamical partition of the circle}

Given a circle homeomorphism $f$ with irrational rotation number $\rho$, one may consider a positive marked trajectory (i.e., the positive trajectory of a marked point) $\xi_{i}=f^{i}\left(\xi_{0}\right) \in S^{1}$, where $i \geq 0$, and pick out of it the sequence of the dynamical convergents $\xi_{q_{n}}, n \geq 0$. We will also conventionally use $\xi_{q_{-1}}=\xi_{0}-1$. The well-understood arithmetical properties of 
rational convergents and the combinatorial equivalence between $f$ and linear rotation $f_{\rho}$ imply that the dynamical convergents approach the marked point alternating their order in the following way:

$$
\xi_{q_{-1}}<\xi_{q_{1}}<\xi_{q_{3}}<\cdots<\xi_{q_{2 m+1}}<\cdots<\xi_{0}<\cdots<\xi_{q_{2 m}}<\cdots<\xi_{q_{2}}<\xi_{q_{0}}
$$

For the marked trajectory, we use the notations $I_{0}^{n}=I_{0}^{n}\left(\xi_{0}\right)$ and $I_{i}^{n}=f^{i}\left(I_{0}^{n}\right)$, where $I_{0}^{n}\left(\xi_{0}\right)$ is the $n$th generator interval. It is well known that the set of intervals $\mathbf{P}_{n}=\mathbf{P}_{n}\left(\xi_{0}, f\right)$ with mutually disjoint interiors defined as

$$
\mathbf{P}_{n}=\left\{I_{i}^{n-1}, 0 \leq i<q_{n} ; I_{j}^{n}, 0 \leq j<q_{n-1}\right\}
$$

determines a partition of the circle for any $n$. The partition $\mathbf{P}_{n}$ is called the $n$th dynamical partition of $S^{1}$. Obviously the partition $\mathbf{P}_{n+1}$ is a refinement of the partition $\mathbf{P}_{n}$ : indeed the intervals of order $n$ are members of $\mathbf{P}_{n+1}$ and each interval $I_{i}^{n-1} \in \mathbf{P}_{n}, 0 \leq i<q_{n}$, is partitioned into $k_{n+1}+1$ intervals belonging to $\mathbf{P}_{n+1}$ such that

$$
I_{i}^{n-1}=I_{i}^{n+1} \cup \bigcup_{s=0}^{k_{n+1}-1} I_{i+q_{n-1}+s q_{n}}^{n}
$$

\section{Proof of the main results}

In this section, first we prove few lemmas, and then using these lemmas we give the proof of the main results. Note that the first main theorem is proved in a similar way as that of [10].

Lemma 4.1 Let $f$ be a circle homeomorphism with irrational rotation number satisfying conditions (i)-(iii). Consider a system of intervals $\left\{\left(a_{i}, b_{i}\right) \subset S^{1}, i=1,2, \ldots, k\right\}$. Suppose that this system of intervals covers each point of the set $S^{1} \backslash \bigcup_{i=1}^{n} U_{\epsilon_{i}}\left(x_{i}\right)$ at most $p$ times. Then there exists a constant $C_{2}=C_{2}(p, f)$ which depends on $p$ and $f$ such that the following inequality

$$
\prod_{i=1}^{k} R\left(a_{i}, b_{i} ; f\right) \leq C_{2}
$$

holds.

Proof Since $f$ is a $P$-homeomorphism on the set $S^{1} \backslash \bigcup_{i=1}^{n} U_{\epsilon_{i}}\left(x_{i}\right)$ for any interval $\left(a_{i}, b_{i}\right)$, we have

$$
\left|\log R\left(a_{i}, b_{i} ; f\right)\right| \leq 2 \sup _{x, y \in\left[a_{i}, b_{i}\right]}\left|\log f^{\prime}(x)-\log f^{\prime}(y)\right| \leq 2 \underset{\left[a_{i}, b_{i}\right]}{\operatorname{Var}} \log f^{\prime}
$$

Using this inequality, we get

$$
\left|\log \prod_{i=1}^{k} R\left(a_{i}, b_{i} ; f\right)\right| \leq \sum_{i=1}^{k}\left|\log R\left(a_{i}, b_{i} ; f\right)\right| \leq 2 \sum_{i=1}^{k} \underset{\left[a_{i}, b_{i}\right]}{\operatorname{Var} \log f^{\prime}}
$$


By assumption, the system of intervals $\mathcal{F}:=\left\{\left[a_{i}, b_{i}\right], i=1,2, \ldots, k\right\}$ covers each point of the set $S^{1} \backslash \bigcup_{i=1}^{n} U_{\epsilon_{i}}\left(x_{i}\right)$ at most $p$ times. Now we describe this system of intervals as a union of subsystems of $\mathcal{F}_{j}, j \leq p$, in the following way: first we take $\left[a_{1}, b_{1}\right]$ as an element of $\mathcal{F}_{1}$, then consider the intersection $\left[a_{1}, b_{1}\right] \cap\left[a_{2}, b_{2}\right]$; if this intersection is empty, then we count the interval $\left[a_{2}, b_{2}\right]$ is an element of $\mathcal{F}_{1}$, otherwise we count an element of $\mathcal{F}_{2}$. Next, consider $\mathcal{F}_{1} \cap\left[a_{3}, b_{3}\right]$ (here and below the intersection with each element of $\mathcal{F}_{1}$ is considered); if it is empty, we count $\left[a_{3}, b_{3}\right]$ is an element of $\mathcal{F}_{1}$, otherwise we check the intersection $\mathcal{F}_{2} \cap\left[a_{3}, b_{3}\right]$. Again if $\mathcal{F}_{2} \cap\left[a_{3}, b_{3}\right]$ is empty, we count $\left[a_{3}, b_{3}\right]$ is an element of $\mathcal{F}_{2}$, otherwise we count $\left[a_{3}, b_{3}\right]$ is an element of $\mathcal{F}_{3}$. Continuing this process we get all $\mathcal{F}_{j}, j \leq p$. By construction of subsystems $\mathcal{F}_{j}, j \leq p$ of $\mathcal{F}$, the elements of each subsystem do not intersect with each other and $\mathcal{F}=\bigcup_{j} \mathcal{F}_{j}, j \leq p$. Therefore

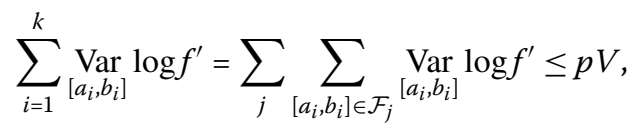

where $V=\operatorname{Var}_{S^{1} \backslash \bigcup_{i=1}^{n} U_{\epsilon_{i}}\left(x_{i}\right)} \log f^{\prime}$.

Lemma 4.2 Let $f$ be a circle homeomorphism with irrational rotation number satisfying conditions (i)-(iii) with one singular point $x_{i^{*}} \in S^{1}$ and corresponding $\alpha_{i^{*}} \in(0,1)$. Then, for any interval $(a, b) \subset S^{1}$, there is a constant $C_{3}=C_{3}(f)$ which depends on $f$ such that

$$
R(a, b ; f)=\frac{(f(b)-f(a))^{2}}{(b-a)^{2}} \cdot \frac{1}{f^{\prime}(a) f^{\prime}(b)} \leq C_{3} .
$$

Proof Denote by $E=S^{1} \backslash\left(x_{i^{*}}-\frac{\epsilon_{i *}}{2}, x_{i^{*}}+\frac{\epsilon_{i}}{2}\right)$ and $V=\left(x_{i^{*}}-\epsilon_{i^{*}}, x_{i^{*}}+\epsilon_{i^{*}}\right)$. Consider the following three cases:

(1) $(a, b) \subset E$;

(2) $(a, b) \subset V$;

(3) neither (1) nor (2).

If $(a, b) \subset E$ then it is easy to get that

$$
R(a, b ; f)=\frac{(f(b)-f(a))^{2}}{(b-a)^{2}} \cdot \frac{1}{f^{\prime}(a) f^{\prime}(b)} \leq \frac{L^{2}}{M^{2}}
$$

where $L=\sup _{x \in E} f^{\prime}(x), M=\inf _{x \in E} f^{\prime}(x)$. Now consider the case $(a, b) \subset V$. We divide this case into two parts:

- the interval $(a, b)$ contains the point $x_{i^{*}}$,

- the interval $(a, b)$ does not contain $x_{i^{*}}$.

If $(a, b)$ does not contain $x_{i^{*}}$, then there can be two cases: $x_{i^{*}} \prec a \prec b$ or $a \prec b \prec x_{i^{*}}$. We prove the first case $x_{i^{*}} \prec a \prec b$, the second case can be analogously proved.

$$
\begin{aligned}
R(a, b ; f) & =\frac{(f(b)-f(a))^{2}}{(b-a)^{2}} \cdot \frac{1}{f^{\prime}(a) f^{\prime}(b)} \\
& =\frac{1}{\alpha_{i^{*}}^{2}} \cdot \frac{\left(\left(b-x_{i^{*}}\right)^{\alpha_{i^{*}}}-\left(a-x_{i^{*}}\right)^{\alpha_{i^{*}}}\right)^{2}}{\left(\left(b-x_{i^{*}}\right)-\left(a-x_{i^{*}}\right)\right)^{2}} \cdot \frac{1}{\left(b-x_{i^{*}}\right)^{\alpha_{i^{*}-1}}\left(a-x_{i^{*}}\right)^{\alpha_{i}-1}} .
\end{aligned}
$$


If we take $y:=\frac{\left(b-x_{i}\right)^{\frac{1}{2}}}{\left(a-x_{i}\right)^{\frac{1}{2}}}$, then it is easy to see that $y>1$, and using this notation we can write (7) in the form

$$
R(y):=R(a, b ; f)=\frac{1}{\alpha_{i^{*}}^{2}} \cdot \frac{\left(y^{\alpha_{i}^{*}}-y^{-\alpha_{i *}}\right)^{2}}{\left(y-y^{-1}\right)^{2}}, \quad y>1
$$

Hence, the function $R(y)=\frac{1}{\alpha_{i^{*}}^{2}} \cdot \frac{\left(y^{\alpha_{i} *}-y^{-\alpha_{i}}\right)^{2}}{\left(y-y^{-1}\right)^{2}}$ is decreasing on $(1, \infty)$ and

$$
\sup _{y>1} R(y)=1
$$

Let $x_{i^{*}} \in(a, b)$, then

$$
\begin{aligned}
R(a, b ; f) & =\frac{(f(b)-f(a))^{2}}{(b-a)^{2}} \cdot \frac{1}{f^{\prime}(a) f^{\prime}(b)} \\
& =\frac{1}{\alpha_{i^{*}}^{2}} \cdot \frac{\left(\left(b-x_{i^{*}}\right)^{\alpha_{i^{*}}}-\left(x_{i^{*}}-a\right)^{\alpha_{i^{*}}}\right)^{2}}{\left(\left(b-x_{i^{*}}\right)-\left(x_{i^{*}}-a\right)\right)^{2}} \cdot \frac{1}{\left(b-x_{i^{*}}\right)^{\alpha_{i} *-1}\left(x_{i^{*}}-a\right)^{\alpha_{i}-1}} .
\end{aligned}
$$

Again if we take $y:=\frac{\left(b-x_{i}\right)^{\frac{1}{2}}}{\left(x_{i^{*}-a}\right)^{\frac{1}{2}}}$, then it is easy to see that $0<y<\infty$, and using this notation we can write (9) in the form

$$
R(y):=R(a, b ; f)=\frac{1}{\alpha_{i^{*}}^{2}} \cdot \frac{\left(y^{\alpha^{*}}+y^{-\alpha_{i}^{*}}\right)^{2}}{\left(y+y^{-1}\right)^{2}}, \quad y>0
$$

Hence, the function $R(y)=\frac{1}{\alpha_{i *}^{2}} \frac{\left(y^{\alpha}+y^{-\alpha_{i}^{*}}\right)^{2}}{\left(y+y^{-1}\right)^{2}}$ is increasing on $(0,1)$ and decreasing on $(1, \infty)$, so

$$
\sup _{0<y<\infty} R(y)=\frac{1}{\alpha_{i^{*}}^{2}}
$$

Now we consider case (3). It is clear that $b-a>\frac{\epsilon_{i} *}{2}$, and since $f$ is strictly increasing we have that $\frac{f(b)-f(a)}{b-a} \leq$ const. Besides that $\frac{1}{f^{\prime}(a) f^{\prime}(b)}$ cannot be sufficiently large. Hence, from this and from (6), (8), (10) the proof of the lemma follows.

Proof of Theorem 2.1 Denote by $E^{*}=S^{1} \backslash \bigcup_{i=1}^{n}\left(x_{i}-\epsilon_{i}, x_{i}+\epsilon_{i}\right), V^{*}=\bigcup_{i=1}^{n}\left(x_{i}-\frac{\epsilon_{i}}{2}, x_{i}+\frac{\epsilon_{i}}{2}\right)$ and $A=\{1,2, \ldots, k\}$. We separate the set $A$ into three disjoint subsets $A_{1}, A_{2}, A_{3}$ as follows:

(a) $A_{1}=\left\{i \in A:\left(a_{i}, b_{i}\right) \subset V^{*}\right\}$,

(b) $A_{2}=\left\{i \in A:\left(a_{i}, b_{i}\right) \subset E^{*}\right\}$,

(c) $A_{3}=A \backslash\left(A_{1} \cup A_{2}\right)$.

Using these subsets we write the left site of (3) in the following form:

$$
\prod_{i=1}^{k} R\left(a_{i}, b_{i} ; f\right)=\prod_{i \in A_{1}} R\left(a_{i}, b_{i} ; f\right) \times \prod_{i \in A_{2}} R\left(a_{i}, b_{i} ; f\right) \times \prod_{i \in A_{3}} R\left(a_{i}, b_{i} ; f\right) .
$$

Now we estimate every product of (11) separately.

(a) Let $i \in A_{1}$. In this case we consider two subsets of $A_{1}: A_{1}^{1}=\left\{i \in A_{1}: x_{j} \in\left(a_{i}, b_{i}\right), j=\right.$ $1,2, \ldots, n\}$ and $A_{1}^{2}=A_{1} \backslash A_{1}^{1}$. Let $i \in A_{1}^{1}$, then by Lemma 4.2 the cross-ratio distortion 
$R\left(a_{i}, b_{i} ; f\right)$ is bounded by $\frac{1}{\alpha_{i}^{2}}$ for fixed $i$ in the neighborhood of each singular point $x_{j}$, $j=1,2, \ldots, n$, and since the number of intervals covering every singular point $x_{j}$ does not exceed $p$, we have

$$
\prod_{i \in A_{1}^{1}} R\left(a_{i}, b_{i} ; f\right) \leq \frac{1}{\alpha_{1}^{2 p}} \cdot \frac{1}{\alpha_{2}^{2 p}} \cdots \frac{1}{\alpha_{n}^{2 p}} .
$$

Let $i \in A_{1}^{2}$, then by Lemma 4.2 we have

$$
\prod_{i \in A_{1}^{2}} R\left(a_{i}, b_{i} ; f\right) \leq 1
$$

(b) Let $i \in A_{2}$, then by Lemma 4.1 we have

$$
\prod_{i \in A_{2}} R\left(a_{i}, b_{i} ; f\right) \leq C_{2}
$$

(c) Let $i \in A_{3}$, then similarly to item (a), the number of intervals covering the end points of $V^{*}$ and $E^{*}$ does not exceed $2 p$, and by Lemma 4.2 we have

$$
\prod_{i \in A_{3}} R\left(a_{i}, b_{i} ; f\right) \leq C_{3}^{2 p n}
$$

Hence, the proof of Theorem 2.1 follows from (11)-(15).

Before we prove the next lemma, we introduce a concept of wandering interval.

Definition 4.3 We say that $I$ is a wandering interval of the circle map $f$ if:

- the intervals $I, f(I), \ldots, f^{n}(I), \ldots$ are pairwise disjoint;

- the $\omega$-limit set of $I$ is not equal to a single periodic orbit.

Lemma 4.4 Suppose that a circle homeomorphism $f$ with irrational rotation number satisfies conditions (i)-(iii) without break points. Then $f$ has no wandering interval.

Proof Assume that $I \subset S^{1}$ is a wandering interval of $f$. It is easy to see that by the properties of dynamical partition of $S^{1}$ and by the mean value theorem we have

$$
\left(f^{q_{n}}(a)\right)^{\prime}=\frac{\left|f^{q_{n}}\left(f^{q_{n-1}}(I)\right)\right|}{\left|f^{q_{n-1}}(I)\right|} \text { and }\left(f^{q_{n}}(b)\right)^{\prime}=\frac{\left|f^{q_{n}}(I)\right|}{|I|},
$$

where $a \in f^{q_{n-1}}(I), b \in I$ and the sign $|\cdot|$ denotes the length of the given interval. If we choose an interval $J=[a, b]$, then $f^{q_{n}}(J)=\left[f^{q_{n}}(a), f^{q_{n}}(b)\right]$. Using (2) and (16) we have

$$
\begin{aligned}
R\left(J ; f^{q_{n}}\right) & =\frac{\left|f^{q_{n}}(J)\right|^{2}}{|J|^{2}} \cdot \frac{1}{\left(f^{q_{n}}(a)\right)^{\prime}\left(f^{q_{n}}(b)\right)^{\prime}} \\
& =\frac{\left|f^{q_{n}}(J)\right|^{2}}{|J|^{2}} \cdot \frac{\left|f^{q_{n-1}}(I)\right|}{\left|f^{q_{n}}(I)\right|} \cdot \frac{|I|}{\mid f^{q_{n}}\left(f^{\left.q_{n-1}(I)\right) \mid}\right.} .
\end{aligned}
$$


Since $I$ is a wandering interval, the sequence $\left\{\left|f^{q_{n}}(I)\right|\right\}_{n=1}^{\infty}$ tends to zero when $n$ goes to infinity, and it follows from this that the second fraction $\frac{f^{q_{n-1}(I) \mid}}{\mid f^{q_{n}(I) \mid}}$ in the second product of (17) does not tend to zero. Since $I \subset f^{q_{n}}(J)$, the first fraction also does not tend to zero, but the third fraction tends to infinity when $n$ goes to infinity, i.e., $\frac{|I|}{\mid f^{q_{n}}\left(f^{\left.q_{n-1}(I)\right) \mid} \rightarrow \infty, n \rightarrow \infty\right.}$. So the last product in (17) tends to infinity. This is a contradiction to (3) and it proves the lemma.

Proof of Theorem 2.2 The proof of the theorem easily follows from Lemma 4.4 with the fact (see [9]) that the homeomorphism $f$ without periodic points is conjugate to the linear rotation $f_{\rho}$ if and only if $f$ does not have a wandering interval.

Competing interests

The authors declare that they have no competing interests.

Authors' contributions

All authors contributed equally to the writing of this paper. All authors read and approved the final manuscript.

\section{Acknowledgements}

The authors would like to acknowledge the financial support received from the Government of Malaysia under the research Grants FRGS/1/2014/ST06/UKM/01/1, DIP-2014-034

Received: 1 April 2014 Accepted: 5 January 2015 Published online: 21 March 2015

\section{References}

1. Cornfeld, IP, Fomin, SV, Sinai, YG: Ergodic Theory. Springer, Berlin (1982)

2. Poincaré, H: Sur les courbes définies par les équations différentielles. J. Math. Pures Appl. (4) 1, 167-244 (1885)

3. Denjoy, A: Sur les courbes définies par les équations différentielles a la surface du tore. J. Math. Pures Appl. (9) 11, 333-375 (1932)

4. Dzhalilov, A, Noorani, S, Akhatkulov, A: On critical circle homeomorphisms with infinite number of break points. Abstr. Appl. Anal. 2014, 378742 (2014)

5. Herman, M: Sur la conjugaison différentiable des difféomorphismes du cercle à des rotations. Publ. Math. Inst. Hautes Études Sci. 49, 5-234 (1979)

6. Świątek, G: On critical circle homeomorphisms. Bol. Soc. Bras. Mat. 29(2), 329-351 (1998)

7. Świątek, G: Circle homeomorphisms with flat critical points. Fundam. Math. 138, 205-217 (1991)

8. Yoccoz, JC: II n'y a pas de contre-exemple de Denjoy analytique. C. R. Acad. Sci. Paris Sér. I Math. 298(7), 141-144 (1984)

9. de Melo, W, van Strien, S: One Dimensional Dynamics. Springer, Berlin (1993)

10. Świątek, G: Rational rotation number for maps of the circle. Commun. Math. Phys. 119(1), 109-128 (1988)

\section{Submit your manuscript to a SpringerOpen ${ }^{\ominus}$ journal and benefit from:}

- Convenient online submission

Rigorous peer review

- Immediate publication on acceptance

- Open access: articles freely available online

- High visibility within the field

- Retaining the copyright to your article 